PREPARED FOR THE U.S. DEPARTMENT OF ENERGY, UNDER CONTRACT DE-AC02-76CH03073

PPPL-3889

PPPL-3889

UC-70

Electromagnetic Weible Instability in intense Charged Particle Beams with Large Energy Anisotropy

by

Edward A. Startsev and Ronald C. Davidson

October 2003

M

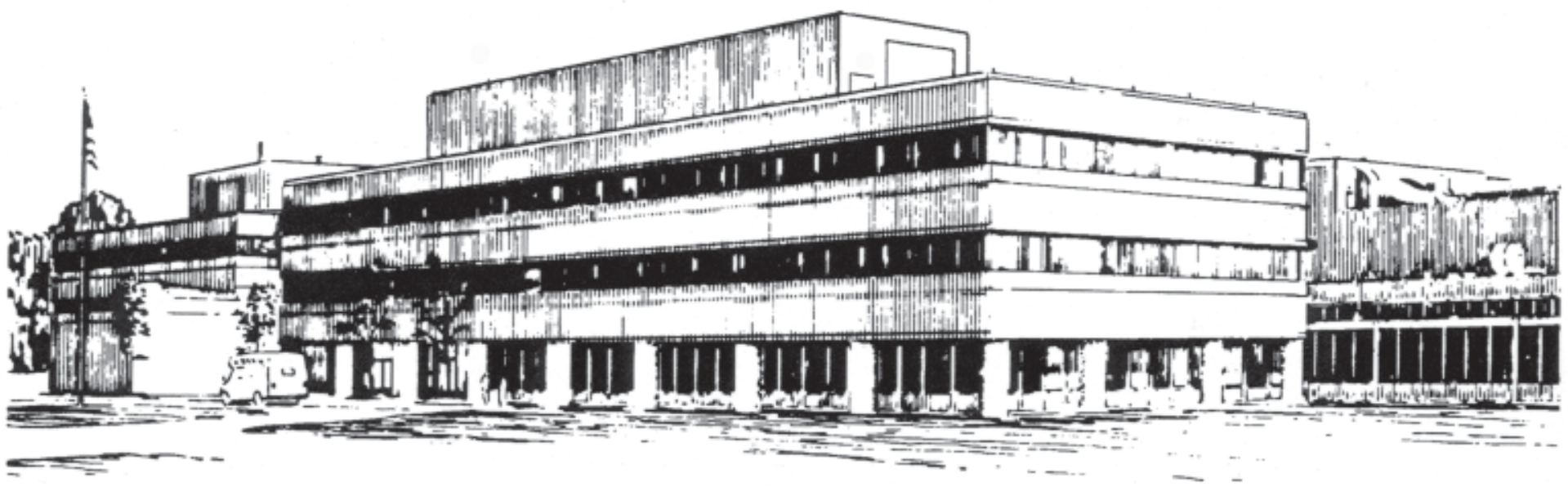

PRINCETON PLASMA PHYSICS LABORATORY PRINCETON UNIVERSITY, PRINCETON, NEW JERSEY 


\section{PPPL Reports Disclaimer}

This report was prepared as an account of work sponsored by an agency of the United States Government. Neither the United States Government nor any agency thereof, nor any of their employees, makes any warranty, express or implied, or assumes any legal liability or responsibility for the accuracy, completeness, or usefulness of any information, apparatus, product, or process disclosed, or represents that its use would not infringe privately owned rights. Reference herein to any specific commercial product, process, or service by trade name, trademark, manufacturer, or otherwise, does not necessarily constitute or imply its endorsement, recommendation, or favoring by the United States Government or any agency thereof. The views and opinions of authors expressed herein do not necessarily state or reflect those of the United States Government or any agency thereof.

\section{Availability}

This report is posted on the U.S. Department of Energy's Princeton Plasma Physics Laboratory Publications and Reports web site in Fiscal Year 2004. The home page for PPPL Reports and Publications is: http://www.pppl.gov/pub_report/

DOE and DOE Contractors can obtain copies of this report from:

U.S. Department of Energy

Office of Scientific and Technical Information

DOE Technical Information Services (DTIS)

P.O. Box 62

Oak Ridge, TN 37831

Telephone: (865) 576-8401

Fax: (865) 576-5728

Email: reports@adonis.osti.gov

This report is available to the general public from:

National Technical Information Service

U.S. Department of Commerce

5285 Port Royal Road

Springfield, VA 22161

Telephone: $1-800-553-6847$ or

(703) $605-6000$

Fax: (703) 321-8547

Internet: http://www.ntis.gov/ordering.htm 


\title{
Electromagnetic Weibel instability in intense charged particle beams with large energy anisotropy
}

\author{
Edward A. Startsev and Ronald C. Davidson \\ Plasma Physics Laboratory, Princeton University, Princeton, New Jersey, 08543
}

\begin{abstract}
In plasmas with strongly anisotropic distribution functions, collective instabilities may develop if there is sufficient coupling between the transverse and longitudinal degrees of freedom. Our previous numerical and theoretical studies of intense charged particle beams with large temperature anisotropy [E. A. Startsev, R. C. Davidson and H. Qin, PRSTAB, 6, 084401 (2003); Phys. Plasmas 9, 3138 (2002)] demonstrated that a fast, electrostatic, Harris-like instability develops, and saturates nonlinearly, for sufficiently large temperature anisotropy $\left(T_{\perp b} / T_{\| b} \gg 1\right)$. The total distribution function after saturation, however, is still far from equipartitioned. In this paper the linearized Vlasov-Maxwell equations are used to investigate detailed properties of the transverse electromagnetic Weibel-type instability for a long charge bunch propagating through a cylindrical pipe of radius $r_{w}$. The kinetic stability analysis is carried out for azimuthally symmetric perturbations about a two-temperature thermal equilibrium distribution in the smooth-focusing approximation. The most unstable modes are identified, and their eigenfrequencies, radial mode structure and instability thresholds are determined. The stability analysis shows that, although there is free energy available to drive the electromagnetic Weibel instability, the finite transverse geometry of the charged particle beam introduces a large threshold value for the temperature anisotropy $\left(\left(T_{\perp b} / T_{\| b}\right)^{\text {Weibel }} \gg\left(T_{\perp b} / T_{\| b}\right)^{\text {Harris }}\right)$ below which the instability is absent. Hence, unlike the case of an electrically neutral plasma, the Weibel instability is not expected to play as significant a role in the process of energy isotropization of intense unneutralized charged particle beams as the electrostatic Harris-type instability.
\end{abstract}




\section{INTRODUCTION}

Periodic focusing accelerators, transport systems and storage rings [1-6] have a wide range of applications ranging from basic scientific research in high energy and nuclear physics, to applications such as heavy ion fusion, spallation neutron sources, tritium production and nuclear waste transmutation, to mention a few examples. Of particular importance at the high beam currents and charge densities of practical interest, are the effects of the intense self fields produced by the beam space charge and current on determining the detailed equilibrium, stability and transport properties. While considerable progress has been made in understanding the self-consistent evolution of the beam distribution function, $f_{b}(\mathbf{x}, \mathbf{p}, t)$, and self-generated electric and magnetic fields, $\mathbf{E}^{s}(\mathbf{x}, t)$ and $\mathbf{B}^{s}(\mathbf{x}, t)$, in kinetic analysis based on the nonlinear Vlasov-Maxwell equations [1, 7-12], in numerical simulation studies of intense beam propagation [13-23], and in macroscopic warm-fluid models [24-27], the effects of finite geometry and space-charge effects often make predictions of detailed stability behavior difficult. It is therefore important to develop an improved understanding of fundamental collective stability properties, including the case where a large temperature anisotropy $\left(T_{\perp b} \gg T_{\| b}\right)$ can drive electrostatic Harris-like [28] and/or electromagnetic Weibel-like [29] instabilities, familiar in the study of electrically neutral plasmas.

It is well known that in neutral plasmas with strongly anisotropic distributions $\left(T_{\| b} / T_{\perp b} \ll\right.$ 1) collective instabilities may develop if there is sufficient coupling between the transverse and longitudinal degrees of freedom [28, 29]. Such anisotropies develop naturally in accelerators. Indeed, due to conservation of energy for particles with charge $e_{b}$ and mass $m_{b}$ accelerated by a voltage $V$, the energy spread of particles in the beam does not change, and (nonrelativistically) $\Delta E_{b i}=m_{b} \Delta v_{b i}^{2} / 2=\Delta E_{b f} \simeq m_{b} V_{b} \Delta v_{b f}$, where $V_{b}=\left(e_{b} V / m_{b}\right)^{1 / 2}$ is the average beam velocity after acceleration. Therefore, the longitudinal velocity spreadsquared, or equivalently the temperature, changes according to $T_{\| b f} \simeq T_{\| b i}^{2} / 2 e_{b} V$ ( for a nonrelativistic beam). At the same time, the transverse temperature may increase due to nonlinearities in the applied and self-field forces, nonstationary beam profiles, and beam mis- 
match. These processes provide the free energy to drive collective instabilities and may lead to a detoriation of beam quality $[22,30,31]$. Such instabilities may also lead to an increase of longitudinal velocity spread, which will make the focusing of the beam difficult and may impose a limit on the minimum spot size achievable in heavy ion fusion experiments.

Previous studies have mostly focused on electrostatic Harris-like anisotropy-driven instability for long, coasting beams $[9,12-14,25,32-36]$. It has been shown that moderately intense beams with normalized beam intensity $s_{b}=\widehat{\omega}_{p b}^{2} / 2 \gamma_{b}^{2} \omega_{f}^{2} \gtrsim 0.5$ are linearly unstable to short-wavelength, perturbations with $k_{z}^{2} r_{b}^{2} \gtrsim 1$, provided the ratio of longitudinal to transverse temperatures $\left(T_{\| b} / T_{\perp b}\right)$ is smaller than some small threshold value. Here, $\widehat{\omega}_{p b}^{2}=4 \pi \widehat{n}_{b} e_{b}^{2} / \gamma_{b} m_{b}$ is the relativistic plasma frequency-squared, $\widehat{n}_{b}$ is the on-axis number density of beam particles, $\gamma_{b}=\left(1-V_{b}^{2} / c^{2}\right)^{-1 / 2}$ is the relativistic mass factor, $V_{b}=\beta_{b} c$ is the average beam velocity in the axial direction and $\omega_{f}=$ const. is the smooth-focusing frequency associated with the applied focusing field. Our recent studies [12-14] have demonstrated that, although the electrostatic, Harris-like instability develops in intense charged particle beams with $T_{\perp b} / T_{\| b} \gg 1$, it saturates for relatively large values of temperature anisotropy, and the total distribution function after saturation is still far from equipartitioned. It is well known in large-size electrically neutral plasmas, that the transverse electromagnetic Weibel instability can occur even for small temperature anisotropy. The purpose of the present paper is to study the Weibel instability mechanism for intense noneutral charged particle beams. While the Weibel instability [29] has been extensively investigated for counterstreaming, anisotropic, electrically neutral plasmas [37], intense electron beam propagation through a large-volume neutralizing plasma background [38-40], for fast ignition schemes where an intense charged particle beam interacts with a dense target plasma [41-45], and for interpenetrating electron-positron plasmas [46], to the best of our knowledge the present analysis represents the first theoretical investigation of the Weibel instability for a one-component, anisotropic $\left(T_{\perp b} \gg T_{\| b}\right)$ charged particle beam, including the important effects of intense space-charge fields.

In Sec. II, a simplified kinetic theory of the Weibel instability is presented for axially 
symmetric $(\partial / \partial \theta=0)$ transverse electromagnetic perturbations about a bi-Maxwellian distribution of beam particles. The analysis leads to the matrix dispersion equation (20) derived from the linearized Vlasov-Maxwell equations, which constitutes a relatively straightforward generalization of the analysis of the Weibel instability for an electrically neutral plasma to the case of a charged particle beam with intense self fields. The most unstable modes are identified, and their eigenfrequencies, and radial mode structure are determined. The instability threshold is obtained, and the relative importance of the Weibel instability and the Harris instability for anisotropic, intense charged particle beams is discussed.

\section{LINEAR STABILITY THEORY}

We briefly outline here a simple derivation of the dispersion relation for the Weibel instability in an intense charged particle beam. The analysis assumes transverse electromagnetic perturbations about a thermal equilibrium distribution with temperature anisotropy $\left(T_{\perp b}>T_{\| b}\right)$ described in the beam frame $\left(V_{b}=0\right.$ and $\left.\gamma_{b}=1\right)$ by the self-consistent axisymmetric Vlasov equilibrium $[1,11]$

$$
f_{b}^{0}(r, \mathbf{p})=\frac{\widehat{n}_{b}}{\left(2 \pi m_{b} T_{\perp b}\right)} \exp \left(-\frac{H_{\perp}}{T_{\perp b}}\right) \frac{1}{\left(2 \pi m_{b} T_{\| b}\right)^{1 / 2}} \exp \left(-\frac{p_{z}^{2}}{2 m_{b} T_{\| b}}\right)
$$

Here, $H_{\perp}=p_{\perp}^{2} / 2 m_{b}+(1 / 2) m_{b} \omega_{f}^{2}\left(x^{2}+y^{2}\right)+e_{b} \phi^{0}(r)$ is the single-particle Hamiltonian for the transverse particle motion, $p_{\perp}=\left(p_{x}^{2}+p_{y}^{2}\right)^{1 / 2}$ is the transverse particle momentum, $r=$ $\left(x^{2}+y^{2}\right)^{1 / 2}$ is the radial distance from the beam axis, $\omega_{f}=$ const. is the transverse frequency associated with the applied focusing field in the smooth-focusing approximation, and $\phi^{0}(r)$ is the equilibrium space-charge potential determined self-consistently from Poisson's equation,

$$
\frac{1}{r} \frac{\partial}{\partial r} r \frac{\partial \phi^{0}}{\partial r}=-4 \pi e_{b} n_{b}^{0}
$$

where $n_{b}^{0}(r)=\int d^{3} p f_{b}^{0}(r, \mathbf{p})$ is the equilibrium number density of beam particles. For simplicity, the analysis is carried out in the beam frame $\left(V_{b}=0\right.$ and $\left.\gamma_{b}=1\right)$. Furthermore, setting $\phi^{0}(r=0)=0$, the constant $\widehat{n}_{b}$ occurring in Eq. (1) can be identified with the on-axis density $n_{b}^{0}(r=0)$, and the constants $T_{\perp b}$ and $T_{\| b}$ can be identified with the transverse and 
longitudinal temperatures (energy units), respectively. Finally, $e_{b}$ and $m_{b}$ are the charge and mass, respectively, of a beam particle.

For present purposes, we consider small-amplitude, axisymmetric $(\partial / \partial \theta=0)$ electromagnetic perturbations of the form

$$
\delta \mathbf{A}(\mathbf{x}, t)=\delta A_{\theta}(\mathbf{x}, t) \mathbf{e}_{\theta}=\mathbf{e}_{\theta} \delta \widehat{A}_{\theta}(r) \exp \left(i k_{z} z-i \omega t\right)
$$

where $\delta \mathbf{A}(\mathbf{x}, t)$ is the perturbed vector potential, $k_{z}$ is the axial wavenumber, and $\omega$ is the complex oscillation frequency, with $\operatorname{Im} \omega>0$ corresponding to instability (temporal growth). Note that we have chosen the Coulomb gauge with $\nabla \cdot \delta \mathbf{A}=0$. The nonzero components of the electromagnetic field perturbations can be expressed in terms of the vector potential as

$$
\begin{array}{r}
\delta E_{\theta}=\frac{i \omega}{c} \delta A_{\theta}, \\
\delta B_{r}=-i k_{z} \delta A_{\theta}, \\
\delta B_{z}=\frac{1}{r} \frac{\partial}{\partial r}\left(r \delta A_{\theta}\right) .
\end{array}
$$

Note that the fields in Eq. (4) are purely electromagnetic with transverse polarization. Indeed, $\nabla \cdot \delta \mathbf{E}=4 \pi e_{b} \delta n_{b}=0$, and there is no charge perturbation, although the current perturbation, $\delta \mathbf{J}_{b}=e_{b} \int d^{3} p \mathbf{v} \delta f_{b}$ is generally non-zero. The linearized Maxwell's equation for $\delta A_{\theta}$ can be expressed as

$$
\frac{\partial}{\partial r} \frac{1}{r} \frac{\partial}{\partial r} r \delta \widehat{A}_{\theta}(r)-k_{z}^{2} \delta \widehat{A}_{\theta}(r)+\frac{\omega^{2}}{c^{2}} \delta \widehat{A}_{\theta}(r)=-\frac{4 \pi e_{b}}{c} \int d^{3} p v_{\theta} \widehat{\delta f_{b}}(r, \mathbf{p})
$$

where $\widehat{\delta f_{b}}(r, \mathbf{p})$ is the Fourier amplitude of the perturbed distribution function $\delta f_{b}(r, z, \mathbf{p}, t)$, i.e.,

$$
\delta f_{b}(r, z, \mathbf{p}, t)=\widehat{\delta f_{b}}(r, \mathbf{p}) \exp \left(i k_{z} z-i \omega t\right)
$$

The perturbed distribution function $\delta f_{b}(r, z, \mathbf{p}, t)$ satisfies the linearized Vlasov equation [1]

$$
\left\{\frac{\partial}{\partial t}+\mathbf{v} \cdot \frac{\partial}{\partial \mathbf{x}}-\left(e_{b} \nabla_{\perp} \phi^{0}+m_{b} \omega_{f}^{2} \mathbf{x}_{\perp}\right) \cdot \frac{\partial}{\partial \mathbf{p}_{\perp}}\right\} \delta f_{b}=-e_{b}\left(\delta \mathbf{E}+\frac{1}{c} \mathbf{v} \times \delta \mathbf{B}\right) \cdot \frac{\partial}{\partial \mathbf{p}} f_{b}^{0}
$$


Using Eqs. (4), we can rewrite the right-hand side of Eq. (7) as

$$
\begin{aligned}
& e_{b}\left(\delta \mathbf{E}+\frac{1}{c} \mathbf{v} \times \delta \mathbf{B}\right) \cdot \frac{\partial}{\partial \mathbf{p}} f_{b}^{0}=\frac{e_{b}\left(x p_{y}-y p_{x}\right)}{m_{b} r}\left\{\delta E_{\theta} \frac{\partial f_{b}^{0}}{\partial H_{\perp}}+\frac{\delta B_{r}}{c}\left(v_{z} \frac{\partial}{\partial H_{\perp}}-\frac{\partial}{\partial p_{z}}\right) f_{b}^{0}\right\} \\
& =\frac{i e_{b}}{m_{b} c} \frac{\delta A_{\theta}}{r} P_{\theta}\left\{\left(\omega-k_{z} v_{z}\right) \frac{\partial f_{b}^{0}}{\partial H_{\perp}}+k_{z} v_{z} \frac{\partial f_{b}^{0}}{\partial H_{\|}}\right\} .
\end{aligned}
$$

Here, $H_{\|}=p_{z}^{2} / 2 m_{b}, v_{z}=p_{z} / m_{b}$, and $P_{\theta}=r p_{\theta}=x p_{y}-y p_{x}=$ const. is the canonical angular momentum of a beam particle moving in the axisymmetric equilibrium configuration. Finally, using the method of characteristics [1], the solution to the linearized Vlasov equation (7) can be expressed as

$$
\begin{array}{r}
\widehat{\delta f_{b}}(r, \mathbf{p})=-\frac{e_{b}}{c m_{b}} P_{\theta}\left[\left(\omega-k_{z} v_{z}\right) \frac{\partial f_{b}^{0}}{\partial H_{\perp}}+k_{z} v_{z} \frac{\partial f_{b}^{0}}{\partial H_{\|}}\right] \\
\times i \int_{-\infty}^{t} d t^{\prime} \frac{\delta \widehat{A}_{\theta}\left[r^{\prime}\left(t^{\prime}\right)\right]}{r^{\prime}\left(t^{\prime}\right)} \exp \left\{i\left(k_{z} v_{z}-\omega\right)\left(t^{\prime}-t\right)\right\}
\end{array}
$$

for perturbations about the choice of the anisotropic thermal equilibrium distribution function in Eq. (1). In the orbit integral in Eq. (9), $\operatorname{Im} \omega>0$ is assumed, and $r^{\prime}\left(t^{\prime}\right)=$ $\left[x^{\prime 2}\left(t^{\prime}\right)+y^{\prime 2}\left(t^{\prime}\right)\right]^{1 / 2}$ is the transverse orbit in the equilibrium field configuration such that $\left[\mathbf{x}_{\perp}^{\prime}\left(t^{\prime}\right), \mathbf{p}_{\perp}^{\prime}\left(t^{\prime}\right)\right]$ passes through the phase-space point $\left(\mathbf{x}_{\perp}, \mathbf{p}_{\perp}\right)$ at time $t^{\prime}=t[1]$. The function $r^{\prime}\left(t^{\prime}\right)$ is a periodic function of $t^{\prime}$ with period $T_{r}=2 \pi / \omega_{r}$, where $\omega_{r}\left(H_{\perp}, P_{\theta}\right)$ is the frequency of radial oscillations. We expand in the Fourier series representation

$$
\frac{\delta \widehat{A}_{\theta}\left[r^{\prime}\left(t^{\prime}\right)\right]}{r^{\prime}\left(t^{\prime}\right)}=\sum_{n} \exp \left[i n \omega_{r}\left(t^{\prime}-t_{\text {min }}^{\prime}\right)\right] \int_{0}^{T_{r}} \frac{d \tau}{T_{r}} \frac{\delta \widehat{A}_{\theta}[\widehat{r}(\tau)]}{\widehat{r}(\tau)} \exp \left\{-i n \omega_{r} \tau\right\}
$$

where $\widehat{r}(\tau)$ is defined by the equation

$$
\tau=\int_{r_{\min }^{\prime}}^{\widehat{r}(\tau)} \frac{d \bar{r}}{\sqrt{2 m_{b}\left[H_{\perp}-P_{\theta}^{2} / 2 m_{b} \bar{r}^{2}-\psi_{0}(\bar{r})\right]}},
$$

and $\psi_{0}(\bar{r})=m_{b} \omega_{f}^{2} \bar{r}^{2} / 2+e_{b} \phi_{0}(\bar{r})$. Here, $t_{\text {min }}^{\prime}$ is defined by $r^{\prime}\left(t_{\text {min }}^{\prime}\right)=r_{\text {min }}^{\prime}\left(H_{\perp}, P_{\theta}\right)$, where $r_{\text {min }}^{\prime}\left(H_{\perp}, P_{\theta}\right)$ is the minimum radial excursion of the particle trajectory undergoing periodic motion. Substituting Eq. (10) into Eq. (9) and integrating over $t^{\prime}$, we obtain

$$
\widehat{\delta f_{b}}(r, \mathbf{p})=-\frac{e_{b}}{m_{b} c} P_{\theta}\left[\left(\omega-k_{z} v_{z}\right) \frac{\partial f_{b}^{0}}{\partial H_{\perp}}+k_{z} v_{z} \frac{\partial f_{b}^{0}}{\partial H_{\|}}\right] \sum_{n} \frac{\exp \left(i n \omega_{r} \hat{t}\right)}{n \omega_{r}+k_{z} v_{z}-\omega} I^{n}\left(H_{\perp}, P_{\theta}\right)
$$


In Eq. (12), the quantity $\widehat{t}$ can be expressed as

$$
\widehat{t}\left(r, H_{\perp}, P_{\theta}\right)=\int_{r_{\min }^{\prime}}^{r} \frac{d \bar{r}}{\sqrt{2 m_{b}\left[H_{\perp}-P_{\theta}^{2} / 2 m_{b} \bar{r}^{2}-\psi_{0}(\bar{r})\right]}} .
$$

Furthermore, $I^{n}\left(H_{\perp}, P_{\theta}\right)$ is defined by

$$
I^{n}\left(H_{\perp}, P_{\theta}\right)=\int_{0}^{T_{r}} \frac{d \tau}{T_{r}} \frac{\widehat{A}_{\theta}[\widehat{r}(\tau)]}{\widehat{r}(\tau)} \exp \left\{-i n \omega_{r} \tau\right\} .
$$

In Eq. (5), we express the perturbation amplitude as $\delta \widehat{A_{\theta}}(r)=\sum_{n} \alpha_{n} A_{n}(r)$, where $\left\{\alpha_{n}\right\}$ are constants, and the complete set of vacuum eigenfunctions $\left\{A_{n}(r)\right\}$ is defined by $A_{n}(r)=C_{n} J_{1}\left(\lambda_{n} r / r_{w}\right)$. Here, $\lambda_{n}$ is the n'th zero of $J_{1}\left(\lambda_{n}\right)=0$, and $C_{n}=1 / r_{w}$ is a normalization constant such that $\int_{0}^{r_{w}} d r r A_{n}(r) A_{n^{\prime}}(r)=\left[J_{2}^{2}\left(\lambda_{n}\right) / 2\right] \delta_{n, n^{\prime}}$. We substitute $\delta \widehat{A_{\theta}}(r)=\sum_{n} \alpha_{n} A_{n}(r)$ into Maxwell's equation (5) and operate with $\int_{0}^{r_{w}} d r r A_{n^{\prime}}(r) \cdots$. This gives the matrix dispersion equation

$$
\sum_{n} \alpha_{n} D_{n, n^{\prime}}(\omega)=0
$$

where $D_{n, n^{\prime}}(\omega)$ is defined by

$$
D_{n, n^{\prime}}(\omega)=\frac{J_{2}^{2}\left(\lambda_{n}\right)}{2}\left(\lambda_{n}^{2}+k_{z}^{2} r_{w}^{2}-r_{w}^{2} \frac{\omega^{2}}{c^{2}}\right) \delta_{n, n^{\prime}}+\chi_{n, n^{\prime}}(\omega),
$$

and the beam-induced susceptibility $\chi_{n, n^{\prime}}(\omega)$ is defined by

$$
\chi_{n, n^{\prime}}(\omega)=-\frac{4 \pi e_{b}}{c} r_{w}^{2} \int_{0}^{r_{w}} d r r A_{n^{\prime}}(r) \int d^{3} p v_{\theta} \widehat{\delta f_{b}^{n}}(r, \mathbf{p})
$$

Here, $\widehat{\delta f_{b}^{n}}(r, \mathbf{p})$ is defined by Eq. (12) with $\delta \widehat{A}_{\theta} \rightarrow A_{n}$.

By changing the integration variables in Eq. (17) from $\left\{r, p_{r}, p_{\theta}\right\}$ to $\left\{\hat{t}, H_{\perp}, P_{\theta}\right\}$, where $\widehat{t}\left(r, H_{\perp}, P_{\theta}\right)$ is the time measured along the particle trajectory from the point where the radial distance is equal to $r_{\text {min }}^{\prime}$ defined in Eq. (13), the integration volume transforms according to $r d r d p_{r} d p_{\theta}=d P_{\theta} d H_{\perp} d \widehat{t}$. Using Eq. (12), it follows that Eq. (17) can be rewritten as

$$
\begin{aligned}
& \chi_{n, n^{\prime}}(\omega)=\frac{\widehat{\omega}_{p b}^{2}}{c^{2}} \sum_{p} \int \frac{d P_{\theta} P_{\theta}^{2}}{m_{b}^{2} \omega_{r}} \frac{d H_{\perp}}{T_{\perp b}^{2}} d p_{z} \exp \left[-\frac{H_{\perp}}{T_{\perp b}}\right] f_{M}\left(p_{z}\right) \\
& \times\left[\frac{\omega-k_{z} v_{z}}{\omega-p \omega_{r}-k_{z} v_{z}}+\frac{k_{z}^{2} T_{\perp b} / m_{b}}{\left(\omega-p \omega_{r}-k_{z} v_{z}\right)^{2}}\right]\left(I_{n}^{p}\right)^{*} I_{n^{\prime}}^{p}
\end{aligned}
$$


where $\widehat{\omega}_{p b}^{2}=4 \pi e_{b}^{2} \widehat{n}_{b} / m_{b}$ is the plasma frequency-squared, and $f_{M}\left(p_{z}\right)=$ $\left(2 \pi m_{b} T_{\| b}\right)^{-1 / 2} \exp \left(-p_{z}^{2} / 2 m_{b} T_{\| b}\right)$. In Eq. (18), $I_{n}^{p}$ is defined by

$$
I_{n}^{p}\left(H_{\perp}, P_{\theta}\right)=\int_{0}^{T_{r}} \frac{d \tau}{T_{r}} \frac{J_{1}\left[\frac{\lambda_{n} \widehat{r}(\tau)}{r_{w}}\right]}{\widehat{r}(\tau)} \exp \left\{-i p \omega_{r} \tau\right\}
$$

In Eq. (18), ( )* denotes complex conjugate. The condition for a nontrivial solution to Eq. $(15)$ is

$$
\operatorname{det}\left\{D_{n, n^{\prime}}(\omega)\right\}=0
$$

which plays the role of a matrix dispersion relation that determines the complex oscillation frequency $\omega$.

In the following analysis, it is convenient to introduce the effective depressed betatron frequency $\omega_{\beta \perp}$. It can be shown [1] that for the equilibrium distribution in Eq. (1), the mean-square beam radius $r_{b}^{2}$ defined by

$$
r_{b}^{2}=\left\langle r^{2}\right\rangle=\frac{\int d r r^{3} n_{b}^{0}(r)}{\int d r r n_{b}^{0}(r)}
$$

is related exactly to the line density $N_{b}=2 \pi \int d r r n_{b}^{0}(r)$, where $n_{b}^{0}(r)=\int d^{3} p f_{b}^{0}(r, \mathbf{p})$, and the transverse beam temperature $T_{\perp b}$ by the equilibrium radial force balance equation [1]

$$
\omega_{f}^{2} r_{b}^{2}=\frac{N_{b} e_{b}^{2}}{m_{b}}+\frac{2 T_{\perp b}}{m_{b}}
$$

Equation (22) can be rewritten as

$$
\left(\omega_{f}^{2}-\frac{1}{2} \bar{\omega}_{p b}^{2}\right) r_{b}^{2}=\frac{2 T_{\perp b}}{m_{b}}
$$

where we have introduced the effective average beam plasma frequency $\bar{\omega}_{p b}$ defined by

$$
r_{b}^{2} \bar{\omega}_{p b}^{2} \equiv \int_{0}^{r_{w}} d r r \omega_{p b}^{2}(r)=\frac{2 e_{b}^{2} N_{b}}{m_{b}} .
$$

Then, Eq. (23) can be used to introduce the effective depressed betatron frequency $\omega_{\beta \perp}$ defined by

$$
\omega_{\beta \perp}^{2} \equiv\left(\omega_{f}^{2}-\frac{1}{2} \bar{\omega}_{p b}^{2}\right)=\frac{2 T_{\perp b}}{m_{b} r_{b}^{2}}
$$


If, for example, the beam density were uniform over the beam cross-section, then Eq. (25) corresponds to the usual definition of the depressed betatron frequency for a KapchinskijVladimirskij $(\mathrm{KV})$ beam equilibrium, and it is readily shown that the radial orbit $\widehat{r}(\tau)$ occurring in Eqs. (11), (14) and (19) can be expressed as

$$
\widehat{r}^{2}(\tau)=\frac{H_{\perp}}{m_{b} \omega_{\beta \perp}^{2}}\left[1-\sqrt{1-\left(\frac{\omega_{\beta \perp} P_{\theta}}{H_{\perp}}\right)^{2}} \cos \left(2 \omega_{\beta \perp} \tau\right)\right] .
$$

In general, for the choice of equilibrium distribution function in Eq. (1), there will be a spread in transverse depressed betatron frequencies $\omega_{\beta \perp}\left(H_{\perp}, P_{\theta}\right)$, and the particle trajectories will not be described by the simple trigonometric function in Eq. (26). For present purposes, however, we consider a simple model in which the radial orbit $\widehat{r}(\tau)$ occurring in Eqs. (11), (14) and (19) is approximated by Eq. (26) with the constant frequency $\omega_{\beta \perp}$ defined in Eq. (25), and the approximate equilibrium density profile is defined by $n_{b}^{0}(r)=\widehat{n}_{b} \exp \left(-m_{b} \omega_{\beta \perp}^{2} r^{2} / 2 T_{\perp b}\right)$. For a nonuniform beam, $\omega_{\beta \perp}^{-1}$ is the characteristic time for a particle with thermal speed $v_{t h \perp}=\left(2 T_{\perp b} / m_{b}\right)^{1 / 2}$ to cross the rms radius $r_{b}$ of the beam. In this case, it is shown in Appendices A and B that $D_{n, n^{\prime}}(\omega)$ can be evaluated in closed analytical form provided the conducting wall is sufficiently far removed from the beam $\left(r_{w} / r_{b} \gtrsim 3\right.$, say). In the limit of an anisotropic beam distribution that is cold in the longitudinal direction, i.e.,

$$
\frac{T_{\| b}}{T_{\perp b}} \rightarrow 0
$$

the result is

$$
\begin{array}{r}
D_{n, n^{\prime}}(\omega)=\frac{J_{2}^{2}\left(\lambda_{n}\right)}{2}\left(\lambda_{n}^{2}+k_{z}^{2} r_{w}^{2}-r_{w}^{2} \frac{\omega^{2}}{c^{2}}\right) \delta_{n, n^{\prime}}+r_{b}^{2} \frac{\widehat{\omega}_{p b}^{2}}{c^{2}} z_{n, n^{\prime}} \exp \left(-z_{n, n}-z_{n^{\prime}, n^{\prime}}\right) \\
\times\left\{\left[I_{0}^{2}\left(z_{n, n^{\prime}}\right)-I_{1}^{2}\left(z_{n, n^{\prime}}\right)\right]\left(1+\frac{k_{z}^{2} v_{t h \perp}^{2}}{2 \omega^{2}}\right)+\sum_{q=1}^{\infty}\left[I_{q}^{2}\left(z_{n, n^{\prime}}\right)-I_{q-1}\left(z_{n, n^{\prime}}\right) I_{q+l}\left(z_{n, n^{\prime}}\right)\right]\right. \\
\left.\times\left(\frac{2 \omega^{2}}{\omega^{2}-4 q^{2} \omega_{\beta \perp}^{2}}+k_{z}^{2} v_{t h \perp}^{2} \frac{\omega^{2}+4 q^{2} \omega_{\beta \perp}^{2}}{\left(\omega^{2}-4 q^{2} \omega_{\beta \perp}^{2}\right)^{2}}\right)\right\} .
\end{array}
$$

Here, $z_{n, n^{\prime}}=\left(r_{b} / r_{w}\right)^{2} \lambda_{n} \lambda_{n^{\prime}} / 4$. In this case, the matrix elements decrease exponentially away from the diagonal, with

$$
\left|\frac{D_{n, n+k}}{D_{n, n}}\right| \sim \exp \left(-\frac{\pi^{2} k^{2}}{4} \frac{r_{b}^{2}}{r_{w}^{2}}\right),
$$


where $k$ is an integer, and we have used the approximate relation $\lambda_{n} \approx \pi(4 n+1) / 4$. Also, because $\widehat{\omega}_{p b}^{2} r_{b}^{2} / c^{2}<<1$ in the parameter regime of practical interest for intense beam applications, the dispersion matrix in Eq. (28) describes modes with frequencies at even multiples of the depressed betatron frequency, i.e., $\omega \approx 2 q \omega_{\beta \perp}$, where $q=0, \pm 1, \pm 2 \ldots$ In the subsequent analysis, we study only the lowest frequency modes with $\omega \approx 0$. Therefore, for $r_{w} / r_{b} \gtrsim 3$, and for the lowest frequency modes, we approximate $\left\{D_{n, n^{\prime}}(\omega)\right\}$ by the finite size square matrix of rank $N$ defined by

$$
\begin{array}{r}
D_{n, n^{\prime}}^{N}(\omega)=\frac{J_{2}^{2}\left(\lambda_{n}\right)}{2}\left(\lambda_{n}^{2}+k_{z}^{2} r_{w}^{2}\right) \delta_{n, n^{\prime}}+r_{b}^{2} \frac{\widehat{\omega}_{p b}^{2}}{c^{2}} z_{n, n^{\prime}} \exp \left(-z_{n, n}-z_{n^{\prime}, n^{\prime}}\right) \\
\times\left\{\left[I_{0}^{2}\left(z_{n, n^{\prime}}\right)-I_{1}^{2}\left(z_{n, n^{\prime}}\right)\right]\left(1+\frac{k_{z}^{2} v_{t h \perp}^{2}}{2 \omega^{2}}\right)\right\},
\end{array}
$$

where $n=1,2, \ldots, N$.

The dispersion matrix (30) can be used to investigate detailed electromagnetic stability properties for strong anisotropy $\left(T_{\| b} / T_{\perp b} \rightarrow 0\right)$ for a wide range of normalized axial wavenumbers $\left(k_{z} r_{b}\right)$ and normalized beam skin depth $\delta_{b}=c / r_{b} \widehat{\omega}_{p b}$. For sufficiently large values of $k_{z} r_{b}$, the large temperature anisotropy $\left(T_{\| b} / T_{\perp b} \rightarrow 0\right)$ in Eq. (30) provides the free energy to drive the classical Weibel-type instability [29], generalized here to include finite transverse geometry and beam space-charge effects. The influence of the finite longitudinal temperature can be taken into account if one assumes $T_{\| b} \neq 0$ in Eq. (1). This results in the (collisionless) growth rate reduction of the unstable mode due to resonant wave-particle interactions [1] associated with the axial momentum spread of the beam particles. The dispersion relation for the case of nonzero longitudinal temperature $T_{\| b} \neq 0$ is derived in Appendix B.

Typical numerical results obtained from the approximate dispersion relation utilizing Eqs. (30) and (B13) are presented in Figs. $1-3$ for the case where $r_{w}=3 r_{b}$. Only the leadingorder terms at frequency $\omega \approx 0$ in Eq. (B13) have been retained in the analysis, and only the results for the most unstable mode are plotted in Figs. $1-3$. Also, for $T_{\| b} / T_{\perp b}=0$, it is found that the growth rate for the instability is nearly independent of the normalized skin depth for $c / r_{b} \widehat{\omega}_{p b}$ in the range $c / r_{b} \widehat{\omega}_{p b} \geq 1$. Therefore, the results in Figs. 1 and 2 are shown for a characteristic value of the normalized skin depth taken to be $c / r_{b} \widehat{\omega}_{p b}=100$. The four curves 
in Figs. 1 and 2 represent the solution obtained using four different values of the dispersion matrix rank $N=2,3,4,5$. It is evident that the curves with $N=4$ and $N=5$ are almost indistinguishable. Figure 1 shows the normalized growth rate $(\operatorname{Im} \omega) /\left(\widehat{\omega}_{p b} v_{\perp b}^{\text {th }} / c\right)$ plotted versus normalized wavenumber $k_{z} r_{b}$ for normalized on-axis beam skin depth $c / r_{b} \widehat{\omega}_{p b}=100$ and temperature ratio $T_{\| b} / T_{\perp b}=0$. Here, $v_{\perp b}^{t h}=\left(2 T_{\perp b} / m_{b}\right)^{1 / 2} \ll c$ is the transverse thermal speed of a beam particle. Because, $|\omega| \sim\left(\widehat{\omega}_{p b} v_{\perp b}^{t h} / c\right) \ll \omega_{\beta \perp}$, the results obtained in Fig. 1 justify the approximation that has been made in the derivation of the dispersion matrix in Eq. (30). Note that the maximum growth rate in Fig. 1 is reduced from the growth rate obtained for an infinite plasma with one active component [29].

The normalized eigenfunction $\delta \widehat{A}_{\theta}(r)$ corresponding to $c / r_{b} \widehat{\omega}_{p b}=100, k_{z} r_{b}=3$ and $T_{|| b} / T_{\perp b}=0$ is plotted versus $r / r_{w}$ in Fig. 2. Note, that the eigenfunction has no zeros (except at $r=0$ and $r=r_{w}$ ) for dispersion matrix ranks $N \geq 3$.

Finally, using Eq. (B13), an important characteristic of the instability, the longitudinal threshold temperature $T_{\| b}^{t h}$ for the onset of instability normalized to the transverse temperature $T_{\perp b}$, is plotted in Fig. 3 versus the normalized skin depth $c / r_{b} \widehat{\omega}_{p b}$. The threshold temperature dependence on the normalized skin depth is very well approximated by the formula $T_{\| b}^{t h} / T_{\perp b} \approx 10^{-0.7} r_{b}^{2} \widehat{\omega}_{p b}^{2} / c^{2}$ [Fig. 3]. For charged particle beams of practical interest $r_{b}^{2} \widehat{\omega}_{p b}^{2} / c^{2} \sim\left(v_{\perp b}^{t h} / c\right)^{2} \ll 1$, and therefore $T_{\| b}^{t h} / T_{\perp b} \ll 1$.

Finally, as noted earlier, for $T_{\| b} / T_{\perp b} \rightarrow 0$ and $r_{b} / r_{w}=1 / 3$, the maximum growth rate in Fig. 1 asymptotes at

$$
(\operatorname{Im} \omega)_{\max }=0.85 \widehat{\omega}_{p b} \frac{v_{\perp b}^{t h}}{c}
$$

for $k_{z} r_{b} \gg 1$, where $\widehat{\omega}_{p b}=\left(4 \pi \widehat{n}_{b} e_{b}^{2} / m_{b}\right)^{1 / 2}$ is the on-axis plasma frequency and $v_{\perp b}^{\text {th }}=$ $\left(2 T_{\perp b} / m_{b}\right)^{1 / 2}$ is the transverse thermal speed. Indeed, a numerical analysis of the dispersion relation shows that the maximum growth estimate in Eq. (31) is valid over the entire range of $\widehat{\omega}_{p b}$ and $v_{\perp b}^{t h}$ consistent with equilibrium force balance in Eq. (23) provided $c / \widehat{\omega}_{p b}>r_{b}$. It is instructive to rewrite Eq. (31) in terms of the average depressed tune $\bar{\nu} / \nu_{0}$ defined by

$$
\frac{\bar{\nu}}{\nu_{0}}=\frac{\omega_{\beta \perp}}{\omega_{f}}=\left(1-\frac{\bar{\omega}_{p b}^{2}}{2 \omega_{f}^{2}}\right)^{1 / 2},
$$


where $\omega_{f}$ is the applied focusing frequency, and $\bar{\omega}_{p b}$ is defined in Eq. (24). For the model density profile $n_{b}^{0}(r)=\widehat{n}_{b} \exp \left(-m_{b} \omega_{\beta \perp}^{2} r^{2} / 2 T_{\perp b}\right)$ assumed in the present analysis, it follows that $\bar{\omega}_{p b}^{2}=\widehat{\omega}_{p b}^{2} / 2$. Making use of Eqs. (25) and (32), and $\widehat{\omega}_{p b}=\sqrt{2} \bar{\omega}_{p b}$ and $r_{b} / r_{w}=1 / 3$, it is straightforward to show that Eq. (31) can be expressed in the equivalent form

$$
\frac{(I m \omega)_{\max }}{\omega_{f}}=0.57 \frac{\omega_{f} r_{w}}{c} \frac{\bar{\nu}}{\nu_{0}}\left(1-\frac{\bar{\nu}^{2}}{\nu_{0}^{2}}\right)^{1 / 2},
$$

where $\omega_{f} r_{w} / c \ll 1$ in the regime of practical interest. Equation (33) displays clearly the dependence of $(\operatorname{Im} \omega)_{\max }$ on the depressed tune, showing that $(\operatorname{Im} \omega)_{\max }$ is a maximum for $\bar{\nu} / \nu_{0}=1 / \sqrt{2}=0.707$ (Fig. 4$)$.

\section{CONCLUSIONS}

To summarize, we have generalized the classical Weibel-type instability to the case of an intense charged particle beam with anisotropic temperature $\left(T_{\| b} / T_{\perp b}<1\right)$ including the important effects of finite transverse geometry and beam space-charge. Using the simplified assumption of negligible spread in depressed betatron frequency $\omega_{\beta \perp}$, we derived a simple dispersion equation for the lowest-order azimuthally-symmetrical $(\partial / \partial \theta=0)$ eigenmode. By numerically solving the matrix dispersion relation, the mode structure, growth rate and condition for the onset of the instability was obtained. It is found that even a small longitudinal temperature, $T_{|| b}^{t h} / T_{\perp b} \approx 10^{-0.7} r_{b}^{2} \widehat{\omega}_{p b}^{2} / c^{2} \sim\left(v_{\perp b}^{t h} / c\right)^{2} \ll 1$, is large enough to stabilize the Weibel instability. The presence of this threshold is due to the finite transverse geometry of the charged particle beam. Indeed, the stabilization criteria for an infinite-size one-component plasma is determined from $T_{\|} / T_{\perp}<1 /\left(1+k^{2} c^{2} / \widehat{\omega}_{p b}^{2}\right)[2]$. In the case of finite transverse geometry, we estimate $k_{\text {min }}^{2} \sim 1 / r_{b}^{2}$ and recover the threshold condition in Fig. 3 in the limit $c^{2} / \widehat{\omega}_{p b}^{2} r_{b}^{2} \gg 1$ up to a numerical factor of order unity specific to the cylindrical geometry. In previous work [12-14] we have shown that the fast, electrostatic, Harris-like

instability develops and saturates at a much larger longitudinal temperature $T_{\| b}^{t h} / T_{\perp b} \approx 0.1$. Hence, we conclude that the Weibel instability is not likely to play a significant role in the process of energy isotropization in intense unneutralized charged particle beams. 
With regard to future investigations, we plan to carry out detailed kinetic simulations of the Weibel instability employing a version of the BEST code $[1,13,17]$ modified to include transverse electromagnetic effects by incorporating a Darwin model [see, for example, Ref. 37] that neglects the displacement current. This will permit detailed investigations of the both the linear and nonlinear phases of the instability; the effects of closer proximity of the conducting wall, where the dispersion matrix defined in Eq. (28) becomes even denser in terms of the importance of the off-diagonal elements; the (stabilizing) influence of a longitudinal momentum spread; and the effects of other choices of distribution function, e.g., a waterbag equilibrium [1].

\section{ACKNOWLEDGEMENTS}

This research was supported by the U. S. Department of Energy. It is a pleasure to acknowledge the benefit of useful discussions with Igor Kaganovich and Wei-li Lee.

\section{APPENDIX A: EVALUATION OF THE ORBIT INTEGRAL}

In this Appendix, we evaluate the orbit integral [see Eq. (19)]

$$
I_{n}^{p}=\int_{0}^{T_{r}} \frac{d \tau}{T_{r}} \frac{J_{1}\left[\lambda_{n} \widehat{r}(\tau) / r_{w}\right]}{\widehat{r}(\tau)} \exp \left[-i p \omega_{r} \tau\right]
$$

where $\widehat{r}(\tau)$ is the trajectory of a particle with transverse energy $H_{\perp}$ and canonical angular momentum $P_{\theta}$ moving in the quadratic potential $\psi(r)=m_{b} \omega_{\beta \perp}^{2} r^{2} / 2$ with initial condition $\widehat{r}(\tau=0)=r_{\text {min }}\left(H_{\perp}, P_{\theta}\right)$ [see Eq. (11)]. Here, $\omega_{r}\left(H_{\perp}, P_{\theta}\right)$ is the frequency of radial motion. In Cartesian coordinates, the particle trajectory $\{\widehat{x}(\tau), \widehat{y}(\tau)\}$ can be expressed as

$$
\begin{aligned}
\widehat{x}(\tau)= & \left.\frac{\sqrt{H_{\perp} / m_{b}}}{\omega_{\beta \perp}} \cos \left(\omega_{\beta \perp} \tau\right)[\cos (\beta)-\sin (\beta))\right], \\
\widehat{y}(\tau) & =\frac{\sqrt{H_{\perp} / m_{b}}}{\omega_{\beta \perp}} \sin \left(\omega_{\beta \perp} \tau\right)[\sin (\beta)+\cos (\beta)],
\end{aligned}
$$

where $\cos (2 \beta)=P_{\theta} \omega_{\beta \perp} / H_{\perp}$. Therefore, we can express

$$
\widehat{r}^{2}(\tau)=\widehat{x}^{2}(\tau)+\widehat{y}^{2}(\tau)=\frac{H_{\perp}}{m_{b} \omega_{\beta \perp}^{2}}\left[1-\sin (2 \beta) \cos \left(2 \omega_{\beta \perp} \tau\right)\right]
$$


From Eq. (A3) it follows that $\omega_{r}\left(H_{\perp}, P_{\theta}\right)=2 \omega_{\beta \perp}$. Substituting Eqs. (A3) into Eq. (A1) and changing the integration variable to $\alpha=2 \omega_{\beta \perp} \tau$, we obtain

$$
F(a, b)=\frac{r_{w}}{\lambda_{n}} I_{n}^{p}=\int_{0}^{2 \pi} \frac{d \alpha}{2 \pi} \frac{J_{1}\left(\sqrt{a^{2}+b^{2}-2 a b \cos (\alpha)}\right)}{\sqrt{a^{2}+b^{2}-2 a b \cos (\alpha)}} \exp [-i p \alpha] .
$$

Here, $a=\left(\lambda_{n} / r_{w} \omega_{\beta \perp}\right) \sqrt{\left(H_{\perp}-\omega_{\beta \perp} P_{\theta}\right) / 2 m_{b}}$ and $b=\left(\lambda_{n} / r_{w} \omega_{\beta \perp}\right) \sqrt{\left(H_{\perp}+\omega_{\beta \perp} P_{\theta}\right) / 2 m_{b}}$. To evaluate this integral we first evaluate

$$
G(a, b)=\int_{0}^{2 \pi} \frac{d \alpha}{2 \pi} J_{0}\left(\sqrt{a^{2}+b^{2}-2 a b \cos (\alpha)}\right) \exp [-i p \alpha]
$$

To evaluate the integral in Eq. (A5), we use the summation theorem for Bessel functions [47], which gives

$$
J_{0}\left(w \sqrt{r^{2}+\rho^{2}-2 r \rho \cos \alpha}\right)=\sum_{k=-\infty}^{+\infty} J_{k}(w \rho) J_{k}(w r) \exp (i k \alpha)
$$

Substituting Eq. (A6) into Eq. (A5), and integrating, we obtain

$$
G(a, b)=J_{p}(a) J_{p}(b)
$$

Next, we use Eq. (A5) to construct the combination

$$
a \frac{\partial G(a, b)}{\partial a}-b \frac{\partial G(a, b)}{\partial b}=\left(b^{2}-a^{2}\right) F(a, b)
$$

Where we have used the fact that $J_{0}^{\prime}(x)=-J_{1}(x)$. Finally, using Eqs. (A7) and (A8), and the recursion relation for Bessel functions, $x J_{n}^{\prime}(x)=-n J_{n}(x)+x J_{n-1}$, we obtain

$$
I_{n}^{p}=\frac{\lambda_{n}}{r_{w}} \frac{a J_{n-1}(a) J_{n}(b)-b J_{n-1}(b) J_{n}(a)}{b^{2}-a^{2}} .
$$

\section{APPENDIX B: EVALUATION OF THE SUSCEPTIBILITY INTEGRAL}

To complete the evaluation of the susceptibility defined in Eq. (18), we determine the following integral

$$
I=\frac{\widehat{\omega}_{p b}^{2}}{c^{2}} \int_{0}^{\infty} \frac{d H_{\perp}}{m_{b}^{2} T_{\perp b}^{2}} \int_{-H_{\perp} / \omega_{\beta}}^{H_{\perp} / \omega_{\beta \perp}} \frac{P_{\theta}^{2} d P_{\theta}}{2 \omega_{\beta \perp}} \exp \left[-\frac{H_{\perp}}{T_{\perp b}}\right]\left(I_{n}^{p}\right)^{*} I_{n^{\prime}}^{p},
$$


where the orbit integral $I_{n^{\prime}}^{p}$ is defined in Eq. (19) and was evaluated in Appendix A. Using Eqs. (19) and (A9), and introducing the change of integration variables,

$$
\begin{gathered}
x=\left(H_{\perp}+P_{\theta} \omega_{\beta \perp}\right) / 2 T_{\perp b}, \\
y=\left(H_{\perp}-P_{\theta} \omega_{\beta \perp}\right) / 2 T_{\perp b},
\end{gathered}
$$

Eq. (B1) can be rewritten as

$$
\begin{aligned}
& I=\frac{T_{\perp b}}{m_{b} c^{2}}\left(\frac{\widehat{\omega}_{p b}}{\omega_{\beta \perp}}\right)^{2} \int_{0}^{\infty} d x \int_{0}^{\infty} d y \exp (-x-y) \\
& \times\left[\sqrt{x} J_{p-1}\left(k_{n} \sqrt{x}\right) J_{p}\left(k_{n} \sqrt{y}\right)-\sqrt{y} J_{p-1}\left(k_{n} \sqrt{y}\right) J_{p}\left(k_{n} \sqrt{x}\right)\right] \\
& \times\left[\sqrt{x} J_{p-1}\left(k_{m} \sqrt{x}\right) J_{p}\left(k_{m} \sqrt{y}\right)-\sqrt{y} J_{p-1}\left(k_{m} \sqrt{y}\right) J_{p}\left(k_{m} \sqrt{x}\right)\right]
\end{aligned}
$$

where $k_{n}=\lambda_{n} \sqrt{T_{\perp b} / m_{b}} / r_{w} \omega_{\beta \perp}$. The change of variables in Eq. (B2) reduces the twodimensional integral in Eq. (B1) to the products of two one-dimensional integrals. The integral in Eq. (B3) can be rewritten as

$$
I=2 \frac{T_{\perp b}}{m_{b} c^{2}}\left(\frac{\widehat{\omega}_{p b}}{\omega_{\beta \perp}}\right)^{2}\left(I_{1}^{n, m} I_{2}^{n, m}-I_{3}^{n, m} I_{3}^{m, n}\right),
$$

where

$$
\begin{gathered}
I_{1}^{n, m}=\int_{0}^{\infty} d x x J_{p-1}\left(k_{n} \sqrt{x}\right) J_{p-1}\left(k_{m} \sqrt{x}\right) \exp (-x), \\
I_{2}^{n, m}=\int_{0}^{\infty} d x J_{p}\left(k_{n} \sqrt{x}\right) J_{p}\left(k_{m} \sqrt{x}\right) \exp (-x)=\exp \left(-\frac{k_{n}^{2}+k_{m}^{2}}{4}\right) I_{p}\left(\frac{k_{n} k_{m}}{2}\right), \\
I_{3}^{n, m}=\int_{0}^{\infty} d x \sqrt{x} J_{p-1}\left(k_{n} \sqrt{x}\right) J_{p}\left(k_{m} \sqrt{x}\right) \exp (-x),
\end{gathered}
$$

We now use the Bessel function identity, $x J_{p-1}(x)=p J_{p}(x)+x J_{p}^{\prime}(x)$, to express the integrals $I_{1}^{n, m}$ and $I_{3}^{n, m}$ in terms of $I_{2}^{n, m}$. This gives

$$
I_{3}^{n, m}=\left(\frac{d}{d k_{n}}+\frac{p}{k_{n}}\right) I_{2}^{n, m}
$$

and

$$
I_{1}^{n, m}=\left(\frac{d}{d k_{n}}+\frac{p}{k_{n}}\right)\left(\frac{d}{d k_{m}}+\frac{p}{k_{m}}\right) I_{2}^{n, m} .
$$


Substituting Eqs. (B6), (B8) and (B9) into Eq. (B4), we obtain after some algebraical manipulation

$$
\begin{aligned}
& I=2 \frac{T_{\perp b}}{m_{b} c^{2}}\left(\frac{\widehat{\omega}_{p b}}{\omega_{\beta \perp}}\right)^{2}\left(I_{2}^{n, m} \frac{\partial^{2}}{\partial k_{n} \partial k_{m}} I_{2}^{n, m}-\frac{\partial}{\partial k_{n}} I_{2}^{n, m} \frac{\partial}{\partial k_{n}} I_{2}^{m, n}\right) \\
& =2 \frac{T_{\perp b}}{m_{b} c^{2}}\left(\frac{\widehat{\omega}_{p b}}{\omega_{\beta \perp}}\right)^{2}\left(\frac{k_{n} k_{m}}{4}\right) \exp \left(-\frac{k_{n}^{2}+k_{m}^{2}}{2}\right) \\
& \times\left[I_{p}^{2}\left(\frac{k_{n} k_{m}}{2}\right)-I_{p-1}\left(\frac{k_{n} k_{m}}{2}\right) I_{p+1}\left(\frac{k_{n} k_{m}}{2}\right)\right],
\end{aligned}
$$

where use has been made of the following identities to eliminate the derivatives of the Bessel functions

$$
\begin{array}{r}
I_{p}^{\prime \prime}(x)+\frac{I_{p}^{\prime}(x)}{x}-\left(1+\frac{p^{2}}{x^{2}} I_{p}(x)\right)=0, \\
x I_{p}^{\prime}(x)=x I_{p-1}(x)-p I_{p}(x), \\
x I_{p}^{\prime}(x)=x I_{p+1}(x)+p I_{p}(x) .
\end{array}
$$

In Eq. (B3), we have extended the integration limits to infinity. The error in this approximation is proportional to $\exp \left[-\left(r_{w} / r_{b}\right)^{2}\right]$, which is negligibly small for $r_{w} / r_{b} \gtrsim 3$. Collecting the result from Eq. (B10), the expression for the matrix elements becomes

$$
\begin{aligned}
& D_{n, n^{\prime}}(\omega)=\frac{J_{2}^{2}\left(\lambda_{n}\right)}{2}\left(\lambda_{n}^{2}+k_{z}^{2} r_{w}^{2}-r_{w}^{2} \frac{\omega^{2}}{c^{2}}\right) \delta_{n, n^{\prime}}+r_{b}^{2} \frac{\widehat{\omega}_{p b}^{2}}{c^{2}} z_{n, n^{\prime}} \exp \left(-z_{n n}-z_{n^{\prime} n^{\prime}}\right)\left\{\left[I_{0}^{2}\left(z_{n n^{\prime}}\right)\right.\right. \\
& \left.-I_{1}^{2}\left(z_{n n^{\prime}}\right)\right] \int d p_{z} f_{0}\left(p_{z}\right)\left(1+\frac{k_{z}^{2} v_{t h \perp}^{2}}{2\left(\omega-k_{z} v_{z}\right)^{2}}\right)+\sum_{q>0}\left[I_{q}^{2}\left(z_{n n^{\prime}}\right)-I_{q+1}\left(z_{n n^{\prime}}\right) I_{q-1}\left(z_{n n^{\prime}}\right)\right] \\
& \left.\times \int d p_{z} f_{0}\left(p_{z}\right)\left(\frac{2\left(\omega-k_{z} v_{z}\right)^{2}}{\left(\omega-k_{z} v_{z}\right)^{2}-4 q^{2} \omega_{\beta \perp}^{2}}+k_{z}^{2} v_{t h \perp}^{2} \frac{\left(\omega-k_{z} v_{z}\right)^{2}+4 q^{2} \omega_{\beta \perp}^{2}}{\left[\left(\omega-k_{z} v_{z}\right)^{2}-4 q^{2} \omega_{\beta \perp}^{2}\right]^{2}}\right)\right\},
\end{aligned}
$$

where $r_{b}^{2}=2 T_{b \perp} / m_{b} \omega_{\beta \perp}^{2}, \quad v_{t h \perp}^{2}=2 T_{\perp b} / m_{b}$, and we have introduced $z_{n, n^{\prime}}=$ $\left(r_{b} / r_{w}\right)^{2} \lambda_{n} \lambda_{n^{\prime}} / 4$. If the longitudinal distribution function $f_{0}\left(p_{z}\right)$ is Maxwellian with $f_{0}\left(p_{z}\right)=$ $\left(2 \pi m_{b} T_{\| b}\right)^{-1 / 2} \exp \left(-p_{z}^{2} / 2 m_{b} T_{\| b}\right)$, then the matrix elements in Eq. (B12) take the form

$$
\begin{array}{r}
D_{n, n^{\prime}}(\omega)=\frac{J_{2}^{2}\left(\lambda_{n}\right)}{2}\left(\lambda_{n}^{2}+k_{z}^{2} r_{w}^{2}-r_{w}^{2} \frac{\omega^{2}}{c^{2}}\right) \delta_{n, n^{\prime}}+r_{b}^{2} \frac{\widehat{\omega}_{p b}^{2}}{c^{2}} z_{n, n^{\prime}} \exp \left(-z_{n n}-z_{n^{\prime} n^{\prime}}\right) \\
\times\left\{\left[I_{0}^{2}\left(z_{n n^{\prime}}\right)-I_{1}^{2}\left(z_{n n^{\prime}}\right)\right] A_{0}(\omega)+\sum_{q>0}\left[I_{q}^{2}\left(z_{n n^{\prime}}\right)-I_{q-1}\left(z_{n n^{\prime}}\right) I_{q+1}\left(z_{n n^{\prime}}\right)\right]\right. \\
\left.\times\left[A_{2 q}(\omega)+A_{-2 q}(\omega)\right]\right\} .
\end{array}
$$


Here, $A_{n}(\omega)$ is defined by

$$
A_{n}(\omega)=\left(1-\frac{v_{t h \perp}^{2}}{v_{t h \|}^{2}}\right)-Z\left(\frac{\omega-n \omega_{\beta \perp}}{k_{z} v_{t h \|}}\right)\left[\frac{\omega}{k_{z} v_{t h \|}}-\frac{\omega-n \omega_{\beta \perp}}{k_{z} v_{t h \|}}\left(1-\frac{v_{t h \perp}^{2}}{v_{t h \|}^{2}}\right)\right],
$$

where $Z(\Omega)$ is the plasma dispersion function $[1,48]$, and $v_{t h \|}=\left(2 T_{\| b} / m_{b}\right)^{1 / 2}$. 


\section{REFERENCES}

[1] R. C. Davidson and H. Qin, Physics of Intense Charged Particle Beams in High Energy Accelerators (World Scientific, Singapore, 2001), and references therein.

[2] R. C. Davidson, Theory of Nonneutral Plasma (Addison-Wesley, Redwood City, CA, 1989).

[3] M. Reiser, Theory and Design of Charged Particle Beams (John Wiley \& Sons, Inc., New York, 1994).

[4] A. W. Chao, Physics of Collective Beam Instabilities in High Energy Accelerators (John Wiley \& Sons, Inc., New York, 1993).

[5] See, for example, Proceedings of the 1999 International Heavy Ion Fusion Symposium, Nuclear Instruments and Methods in Physics Research A, pp. 1-674 (2001).

[6] See, for example, Proceedings of the 2001 Particle Accelerator Conference (IEEE, Piscataway, NJ, 2001), pp. 1-4098.

[7] I. M. Kapchinskij and V. V. Vladimirskij, in Proceedings of the International Conference on High Energy Accelerators and Instrumentation (CERN Scientific Information Service, Geneva, 1959), p.274.

[8] R. L. Gluckstern, in Proceedings of the 1970 Proton Linear Accelerator Conference, Batavia, IL, edited by M. R. Tracy (National Accelerator Laboratory, Batavia, IL, 1971), p. 811.

[9] T. -S. Wang and L. Smith, Particle Accelerators 12, 247 (1982).

[10] R. C. Davidson, Phys. Rev. Lett. 81, 991 (1998).

[11] R. C. Davidson and H. Qin, Physical Review Special Topics on Accelerators and Beams 2, $114401(1999)$.

[12] E. A. Startsev, R. C. Davidson and H. Qin, Physical Review Special Topics on Accelerators and Beams 6, 084401 (2003).

[13] E. A. Startsev, R. C. Davidson and H. Qin, Phys. Plasmas 9, 3138 (2002).

[14] E. A. Startsev, R. C. Davidson and H. Qin, Laser and Particle Beams 20, 585 (2002).

[15] Q. Qian, W. W. Lee, and R. C. Davidson, Phys. Plasmas 4, 1915 (1997).

[16] W. W. Lee, Q. Qian, and R. C. Davidson, Physics Letters A230, 347 (1997).

[17] H. Qin, R. C. Davidson and W. W. Lee, Physical Review Special Topics on Accelerators and Beams 3, 084401 (2000); 3, 109901 (2000).

[18] P. H. Stoltz, R. C. Davidson, and W. W. Lee, Phys. Plasmas 6, 298 (1999). 
[19] H. Qin, R. C. Davidson, W. W. Lee and E. A. Startsev, Proceedings of the 2001 Particle Accelerator Conference (IEEE, Piscataway, NJ, 2001), p. 693.

[20] I. Hofmann, L. J. Laslett, L. Smith and I. Haber, Particle Accelerators 13, 145 (1983).

[21] E. A. Startsev, R. C. Davidson, W. W. Lee and H. Qin, Proceedings of the 2001 Particle Accelerator Conference (IEEE, Piscataway, NJ, 2001), p. 3081.

[22] S. M. Lund, J. J. Barnard, G. D. Craig, A. Friedman, D. P. Grote, T. S. Sangster, W. M. Sharp, S. Eylon, T. T. Fessenden, E. Henestroza, S. Yu, and I. Haber, Nuclear Instruments and Methods in Physics Research A 415, 345 (1998).

[23] A. Friedman, J. J. Barnard, D. P. Grote, and I. Haber, Nuclear Instruments and Methods in Physics Research A 415, 455 (1998).

[24] S. M. Lund and R. C. Davidson, Phys. Plasmas 5, 3028 (1998).

[25] S. Strasburg and R. C. Davidson, Physics Letters A269, 40 (2000).

[26] I. Hofmann, Phys. Rev. E57, 4713 (1998).

[27] R. C. Davidson and S. Strasburg, Phys. Plasmas 7, 2657 (2000).

[28] E. G. Harris, Phys. Rev. Lett. 2, 34 (1959).

[29] E. S. Weibel, Phys. Rev. Lett. 2, 83 (1959).

[30] I. Haber, private communication (2003).

[31] A. Friedman, private communication (2003).

[32] A. Friedman, D. A. Callahan, D. P. Grote, and A. B. Langdon, Bull. Am. Phys. Soc. 35, 2121 (1990).

[33] A. Friedman, R. O. Bangerter, D. A. Callahan, D. P. Grote, and A. B. Langdon, Proceedings of the 2nd European Particle Accelerator Conference, 1699 (1990), edited by P. A. Martin, Vol 2.

[34] A. Friedman, D. P. Grote and I. Haber, Phys. Fluids B 4, 2203 (1992).

[35] S. M. Lund, D. A. Callahan, A. Friedman, D. P. Grote, I. Haber, and T. F. Wang, Proceedings of XIX International Linear Accelerator Conference Chicago, 1998, edited by C. E. Eyberger, R. C. Pardo and M. M. White (Argone National Laboratory, Argone, Illinois, 1998). p.372.

[36] I. Haber, A. Friedman, D. P. Grote, S. M. Lund, and R. A. Kishek, Phys. Plasma 6, 2254 (1999).

[37] R. C. Davidson, D. A. Hammer, I. Haber and C. E. Wagner, Phys. Fluids 15, 317 (1972).

[38] R. Lee and M. Lampe, Phys. Rev. Lett. 31, 1390 (1973).

[39] C. A. Kapetanakos, Appl. Phys. Lett. 25, 484 (1974). 
[40] R. C. Davidson, B. H. Hui and C. A. Kapetanakos, Phys. Fluids 18, 1040 (1975).

[41] Y. Sentoku, K. Mima, P. Kaw, K. Nishikawa, Phys. Rev. Lett. 90, 155001 (2003).

[42] T. Taguchi, T. M. Antonsen, C. S. Liu, K. Mima, Phys. Rev. Lett., 86, 5055 (2001).

[43] M. Honda, J. Meyer-ter-Vehn, A. Pukhov, Phys. Rev. Lett. 85, 2128 (2000).

[44] M. Honda, J. Meyer-ter-Vehn, A. Pukhov, Phys. Plasmas 7, 1302 (2000).

[45] L. O. Silva, R. A. Fonseca, J. Tonge, W. B. Mori and J. M. Dawson, Phys. Plasmas 9, 2458 (2002).

[46] R. A. Fonseca, L. O. Silva, J. Tonge, R. G. Hemker, J. M. Dawson and W. B. Mori, IEEE Trans. Plasma Science 30, 28 (2002).

[47] I. S. Gradshteyn, and I. M. Ryzhik, Tables of Integrals, Series, and Products (Academic Press, New York, 1965).

[48] NRL Plasma Formulary (Naval Research Laboratory, Washington, D. C., 1998). 


\section{FIGURE CAPTIONS}

Fig.1: Plot of the normalized growth rate $(\operatorname{Im} \omega) /\left(\widehat{\omega}_{p b} v_{\perp b}^{t h} / c\right)$ versus $k_{z} r_{b}$ for normalized skin depth $c / r_{b} \widehat{\omega}_{p b}=100$ and $T_{\| b} / T_{\perp b}=0$ obtained from Eq. (30). The four curves correspond to four different values of the dispersion matrix rank $N=2,3,4,5$.

Fig.2: Plots of the normalized eigenfunction versus $r / r_{w}$ corresponding to $c / r_{b} \widehat{\omega}_{p b}=100$, $k_{z} r_{w}=10$ and $T_{\| b} / T_{\perp b}=0$ obtained from Eq. (30). The four curves correspond to four different values of the dispersion matrix rank $N=2,3,4,5$.

Fig.3: The longitudinal threshold temperature $T_{\| b}^{t h}$ for the onset of instability normalized to the transverse temperature $T_{\perp b}$ is plotted versus the normalized skin depth $c / r_{b} \widehat{\omega}_{p b}$ [Eq. (B13)].

Fig.4: Plot of maximum growth rate $(\operatorname{Im} \omega)_{\max }$ obtained from Eqs. (30) and (33) versus the average depressed tune $\bar{\nu} / \nu_{0}$. 


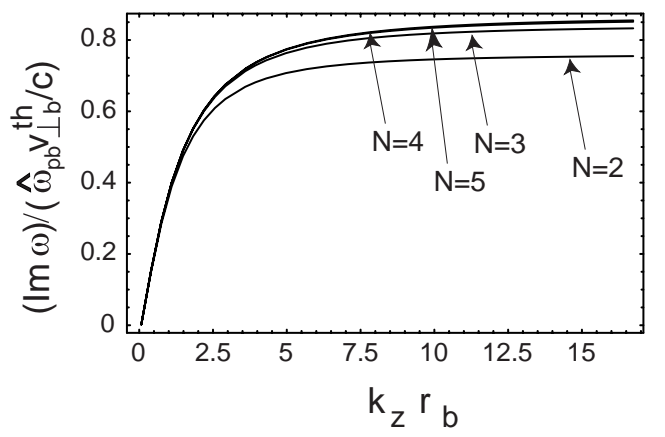

FIG. 1: 


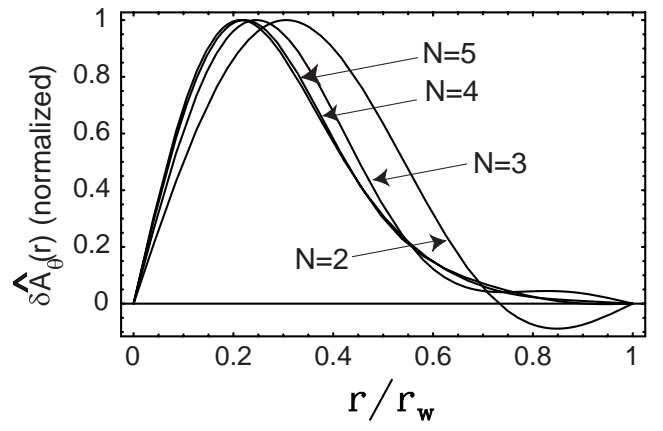

FIG. 2: 


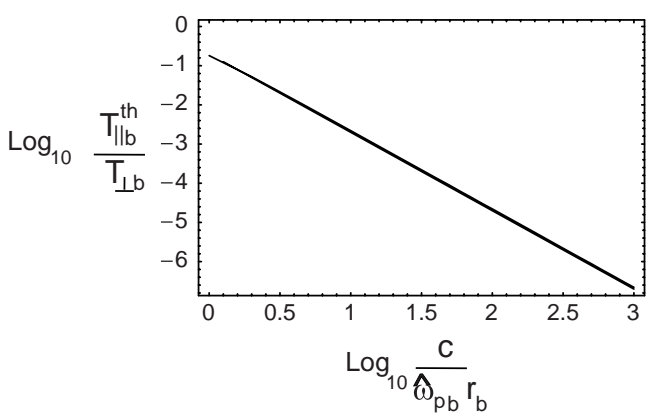

FIG. 3: 


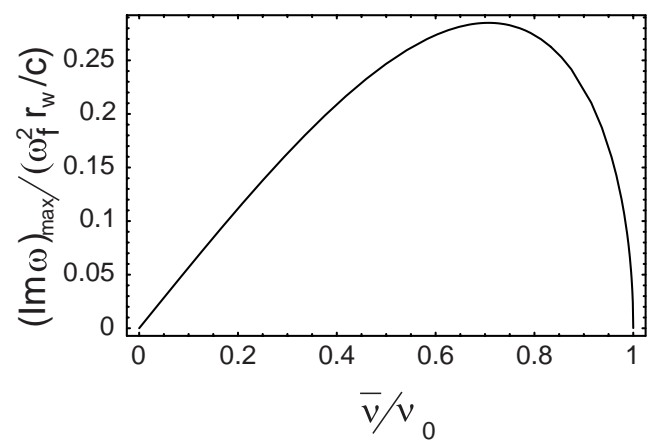

FIG. 4: 


\section{External Distribution}

Plasma Research Laboratory, Australian National University, Australia

Professor I.R. Jones, Flinders University, Australia

Professor João Canalle, Instituto de Fisica DEQ/IF - UERJ, Brazil

Mr. Gerson O. Ludwig, Instituto Nacional de Pesquisas, Brazil

Dr. P.H. Sakanaka, Instituto Fisica, Brazil

The Librarian, Culham Laboratory, England

Mrs. S.A. Hutchinson, JET Library, England

Professor M.N. Bussac, Ecole Polytechnique, France

Librarian, Max-Planck-Institut für Plasmaphysik, Germany

Jolan Moldvai, Reports Library, Hungarian Academy of Sciences, Central Research Institute for Physics, Hungary

Dr. P. Kaw, Institute for Plasma Research, India

Ms. P.J. Pathak, Librarian, Institute for Plasma Research, India

Ms. Clelia De Palo, Associazione EURATOM-ENEA, Italy

Dr. G. Grosso, Instituto di Fisica del Plasma, Italy

Librarian, Naka Fusion Research Establishment, JAERI, Japan

Library, Laboratory for Complex Energy Processes, Institute for Advanced Study, Kyoto University, Japan

Research Information Center, National Institute for Fusion Science, Japan

Dr. O. Mitarai, Kyushu Tokai University, Japan

Dr. Jiangang Li, Institute of Plasma Physics, Chinese Academy of Sciences, People's Republic of China

Professor Yuping Huo, School of Physical Science and Technology, People's Republic of China

Library, Academia Sinica, Institute of Plasma Physics, People's Republic of China

Librarian, Institute of Physics, Chinese Academy of Sciences, People's Republic of China

Dr. S. Mirnov, TRINITI, Troitsk, Russian Federation, Russia

Dr. V.S. Strelkov, Kurchatov Institute, Russian Federation, Russia

Professor Peter Lukac, Katedra Fyziky Plazmy MFF UK, Mlynska dolina F-2, Komenskeho Univerzita, SK-842 15 Bratislava, Slovakia

Dr. G.S. Lee, Korea Basic Science Institute, South Korea

Institute for Plasma Research, University of Maryland, USA

Librarian, Fusion Energy Division, Oak Ridge National Laboratory, USA

Librarian, Institute of Fusion Studies, University of Texas, USA

Librarian, Magnetic Fusion Program, Lawrence Livermore National Laboratory, USA

Library, General Atomics, USA

Plasma Physics Group, Fusion Energy Research Program, University of California at San Diego, USA

Plasma Physics Library, Columbia University, USA

Alkesh Punjabi, Center for Fusion Research and Training, Hampton University, USA

Dr. W.M. Stacey, Fusion Research Center, Georgia Institute of Technology, USA

Dr. John Willis, U.S. Department of Energy, Office of Fusion Energy Sciences, USA

Mr. Paul H. Wright, Indianapolis, Indiana, USA 
The Princeton Plasma Physics Laboratory is operated by Princeton University under contract with the U.S. Department of Energy.

\author{
Information Services \\ Princeton Plasma Physics Laboratory \\ P.O. Box 451 \\ Princeton, NJ 08543
}

Phone: 609-243-2750

Fax: 609-243-2751

e-mail: pppl_info@pppl.gov

Internet Address: http://www.pppl.gov 